\title{
Exogenous Insulin Augments in Healthy Volunteers the Cardiovascular Reactivity to Noradrenaline but Not to Angiotensin II
}

R. O. B. Gans, H. J. G. Bilo, W. W. A. v. Maarschalkerweerd, R. J. Heine, J. J. P. Nauta, and A. J. M. Donker

Department of Medicine, Free University Hospital, Amsterdam, The Netherlands

\begin{abstract}
Hyperinsulinemia has been implicated in the pathogenesis of the blood pressure elevation in patients with noninsulin-dependent diabetes mellitus, obesity, but also essential hypertension. In these conditions an increased cardiovascular reactivity to noradrenaline (NA) and angiotensin II (AII) can be observed. Using the euglycemic clamp technique, we determined the cardiovascular reactivity to graded infusions of NA and AII in nine healthy males before (Bas), and 1 and $6 \mathrm{~h}$ after infusion of insulin ( $50 \mathrm{mU} / \mathrm{kg}$ per $\mathrm{h}$ ) was started. On separate days control experiments were carried out to control for any circadian variation. Insulin led to a decrease of the amount of circulating NA necessary to increase the diastolic blood pressure (DBP) 20 mmHg (actual experiment [mean \pm SEM]: Bas, 23.1 $\pm 5.0 ; 1 \mathrm{~h}$, 14.8 \pm 3.0 ; and $6 \mathrm{~h}, 12.3 \pm 3.1$; and control experiment: Bas, 20.7 $\pm 5.0 ; 1 \mathrm{~h}, 18.6 \pm 3.5$; and $6 \mathrm{~h}, 17.3 \pm 3.3 \mathrm{nmol} / \mathrm{liter}$; Bas vs. 1 and $6 \mathrm{~h}: P<0.05)$. Although the amount of NA infused to raise DBP $20 \mathrm{mmHg}$ showed a similar decline after $1 \mathrm{~h}$ of insulin infusion, no such change from baseline could be observed at $6 \mathrm{~h}$. This appeared to be due to an increase in NA clearance with more prolonged insulin infusion. Insulin exerted no effect on the amount of AII infused to increase DBP $20 \mathrm{mmHg}$ (actual experiment: Bas, 27.6 \pm 6.4 ; 1 h, 28.8 \pm 10.0 ; and 6 h, 21.2 \pm 5.3 ; and control experiment: Bas, 33.6 $\pm 5.7 ; 1 \mathrm{~h}, 34.2 \pm 6.1$; and $6 \mathrm{~h}$, $23.4 \pm 4.7 \mathrm{ng} / \mathrm{kg} / \mathrm{min}$; NS). We did observe a circadian variation in AII reactivity. Whether the increase in cardiovascular responsiveness to NA after administration of insulin contributes to the elevation in blood pressure frequently observed in patients with insulin resistance remains to be proven. ( $J$. Clin. Invest. 1991 . 88:512-518.) Key words: hyperinsulinemia • noradrenaline $\bullet$ angiotensin II $\bullet$ noradrenaline clearance $\bullet$ renin-angiotensin-aldosterone system
\end{abstract}

\section{Introduction}

In patients with borderline or established essential hypertension, an exaggerated cardiovascular reactivity to stimuli such as noradrenaline (NA) ${ }^{1}$ or angiotensin II (AII) has been demon-

Address reprint requests to R. O. B. Gans, M.D., Department of Medicine, Free University Hospital, De Boelelaan 1117, 1081 HV Amsterdam, The Netherlands.

Received for publication 19 September 1990 and in revised form 7 February 1991.

1. Abbreviations used in this paper: Adr, adrenaline; AII, angiotensin II; Ald, aldosterone; DBP, diastolic blood pressure; NA, noradrenaline; PD, pressor dose, PRA, plasma renin activity.

J. Clin. Invest.

(C) The American Society for Clinical Investigation, Inc. 0021-9738/91/08/0512/07 \$2.00

Volume 88, August 1991, 512-518 strated in most (1-3), but not in all studies (4). In the normotensive offspring of hypertensive families, who are at increased risk for the subsequent development of an elevated blood pressure, an enhanced reactivity to NA, but not to AII, can already be shown $(3,5)$. Some experimental data also show that the increase in vascular smooth muscle sensitivity to vasoactive hormones may actually precede or parallel the development of hypertension $(6,7)$.

Recently, the presence of insulin resistance, and as a consequence elevated plasma insulin levels, has been established in patients with untreated essential hypertension and a still normal glucose tolerance according to World Health Organization (WHO) criteria (8). Moreover, a positive relation between basal plasma insulin levels and the height of the blood pressure has been found in a group of nondiabetic subjects, the majority having blood pressure levels within the normal range (9). It is yet unknown if hyperinsulinemia itself contributes to the rise in blood pressure or that hypertension is merely associated with insulin resistance and, therefore, increased plasma insulin levels.

Conflicting data exist about the effect of insulin on cardiovascular reactivity. In in vitro studies an attenuation of the vasoreactivity after insulin administration has been reported $(10,11)$. In the dog both vasodilation and vasoconstriction can be observed during insulin infusion, depending on which vascular bed is studied (10). Also in the dog a low-dose insulin infusion did not affect the blood pressure response to AII (12), whereas with higher dosages a dose-dependent augmentation was observed (13). In the same animal insulin did not appear to affect blood pressure during chronic infusion of NA (14). However, the expected decrease in blood pressure, which occurred during infusion of insulin alone, did not take place, suggestive of some interaction between these hormones.

Studies on the influence of insulin on cardiovascular reactivity in man are scarce. In healthy subjects euglycemic hyperinsulinemia has been reported not to modify the pressor action of AII (15), whereas to our knowledge no such information is available regarding the effect of insulin on NA action. On the other hand, in type-II diabetic patients, who supposedly have high insulin levels and an increased prevalence of hypertension compared with the background population, an increased pressor response to vasoactive hormones can usually be observed (16). Considering these conflicting results and the fact that the cardiovascular response to NA may be dissociated from that to AII, we studied the effect of exogenously administered insulin on the cardiovascular reactivity to NA and AII in normal subjects using the euglycemic clamp technique.

\section{Methods}

Subjects. Nine healthy white males, mean age 26 (range 22-30) yr, were studied. All were normotensive (blood pressure [mean \pm SD]: 123 \pm 6 / $59 \pm 6 \mathrm{mmHg}$ ), within $10 \%$ ideal body weight and taking no medica- 
tion. Their family histories were negative for hypertension and diabetes mellitus. Normal glucose tolerance according to the WHO criteria was confirmed by a 75-g oral glucose tolerance test. Informed consent was given by all subjects and the study had been approved by the local ethical committee.

The week preceding the study all subjects adhered to a $170 \mathrm{mmol}$ sodium and $200 \mathrm{~g}$ carbohydrates containing diet; the extra amount of sodium chloride, necessary in all but one subject, was supplied with capsules containing $500 \mathrm{mg} \mathrm{NaCl}$. Compliance with the diet was confirmed by measurement of the 24-h urinary sodium excretion during the last two days before the study; if the amount of sodium excreted exceeded $150 \mathrm{mmol}$ per day, the study was carried out.

Protocol. All subjects were studied on four occasions, i.e., two insulin-clamp experiments with either noradrenaline or angiotensin II and two control experiments. The sensitivity to either one of the two pressors was assessed three times during the day, i.e., before (Bas), and 1 and $6 \mathrm{~h}$ after an infusion of insulin or insulin solvent (in case of the control experiment) was started. All experiments were carried out in the same room with a controlled ambient temperature of $22^{\circ} \mathrm{C}$.

Insulin-clamp experiments. After an overnight fast all subjects came to the outpatient clinic at $8 \mathrm{am}$. Three polytetrafluoroethylene cannulae (Venflon; Viggo, Helsinborg, Sweden) were inserted. One cannula was inserted retrogradely within a dorsal hand vein and was exclusively used for blood sampling for frequent blood glucose measurements of arterialized blood. Arterialization of venous blood was achieved by maintaining the hand at $\sim 55^{\circ} \mathrm{C}$ in a thermoregulated Plexiglas box (17). Blood glucose measurements in arterialized venous blood have been shown to reflect reliably arterial blood glucose values. Arterial blood glucose values are necessary to ensure stability of the euglycemic hyperinsulinemic clamp and to obtain reliable information regarding insulin sensitivity. Due to a progressive increase in the arteriovenous blood glucose difference with increasing insulin levels (due to increased tissue extraction of glucose), performance of a clamp based on venous blood glucose values would not only increase the arterial blood glucose levels above basal values (with the subsequent stimulation of endogenous insulin release), but also overestimate insulin sensitivity because the latter is derived from the amount of glucose infused to maintain euglycemia. Blood samples for all other determinations but glucose were obtained from a second cannula inserted in an antecubital vein and flushed with $0.9 \%$ sodium chloride after use. A third cannula, in the contralateral antecubital vein, was used for all infusions.

After an equilibration period of $60 \mathrm{~min}$, a baseline pressor infusion (Bas) with either NA or AII was performed (see below). When this was completed, a continuous infusion of insulin (Humulin S; Lilly Co., Indianapolis, IN), diluted to $40 \mathrm{ml}$ with Haemaccel (Hoechst, Amsterdam, The Netherlands), was started at $50 \mathrm{mU} / \mathrm{kg}$ per $\mathrm{h}$ after a priming dose $(0.1 \times \mathrm{kg} \times$ the desired rise in plasma insulin level, i.e., $50 \mathrm{mU} /$ liter) had been given. This would result in blood insulin levels between 50 and $60 \mathrm{mU} /$ liter, a degree of hyperinsulinemia that is still within the physiological range. Fasting blood glucose level was maintained by adjustment of the infusion rate of a $20 \%$ dextrose solution according to a method previously described (18). Blood glucose concentration was measured every $5 \mathrm{~min}$ by an automated glucose oxidase method (Yellow Springs Instruments Co., Yellow Springs, $\mathrm{OH}$ ). 1 and $6 \mathrm{~h}$ after the start of the insulin infusion, the pressor infusion with either NA or AII was repeated. The reason that the pressor infusion was repeated after 6 $h$ of insulin administration was twofold. First, insulin induces a shift of potassium into the cell. This is associated with a hyperpolarization of the cell membrane, making the cell less responsive to stimuli (19). The initial decline in serum potassium tends, however, to become less marked after more prolonged administration of insulin, thereby (partly) eliminating the influence of this possible confounding variable on the outcome of the experiments (20). Second, prolonged administration of insulin is accompanied by an increase in insulin sensitivity (21). This would enable us to study the effect of increasing insulin sensitivity, as opposed to insulin resistance, on the cardiovascular reactivity as well. During the whole study, all subjects were in a supine position, except when voiding.

Cardiovascular reactivity was assessed as previously reported in the literature (2-5). After basal blood pressure (the mean of five measurements) was measured and baseline blood samples were drawn, an infusion with either NA (noradrenaline tartrate, Centrafarm, dissolved in $5 \%$ dextrose) or AII (Hypertensin, Ciba Geigy, dissolved in 5\% dextrose) was begun. Each pressor infusion was started at the lowest infusion rate and progressively increased with predetermined steps (NA: $10,20,40,60,80,100,150,200,250,300,350,400,450$, and 500 $\mathrm{ng} / \mathrm{kg}$ per min; AII: 1, 2, 4, 6, 8, 10, 12, 14, 16, 18, 20, 25, 30, 35, 40, 45, and $50 \mathrm{ng} / \mathrm{kg}$ per $\mathrm{min}$ ). Blood pressure was measured during each infusion rate every minute and the infusion rate was increased to the next one, without a washout period, only after blood pressure had stabilized for at least $5 \mathrm{~min}$. At two infusion rates, that is when increases in diastolic blood pressure of $\sim 10$ and $20 \mathrm{mmHg}$, respectively, were achieved, blood samples were drawn 20 min after starting the corresponding infusion rate for determination of plasma catecholamines (NA and adrenaline [Adr]), plasma renin activity (PRA), and plasma aldosterone (Ald). After such a time-interval plasma levels of both pressor substances, but also plasma Ald, have stabilized (22). Systolic and diastolic blood pressure and heart rate were measured with a semicontinous blood pressure measuring device (Nippon Colin BP $103 \mathrm{~N}$ Sphygmomanometer, Hayashi, Komaki-city, Japan). In addition, heart rhythm was monitored continuously using a unipolar limb lead. The pressor dose (PD) was defined as the amount of the pressor agent required to produce a rise in diastolic blood pressure (DBP) $20 \mathrm{mmHg}$ above baseline and was calculated from the semilogarithmic plot of the individual changes in DBP versus the corresponding NA or AII infusion rates ( $x$-axis: log dose ( $\mathrm{ng} / \mathrm{kg}$ per min); $y$-axis: change in DBP [mmHg]) (23). Likewise, the increase in circulating NA to elevate the DBP $20 \mathrm{mmHg}$ was calculated.

Control experiments. For each pressor hormone separately, a control experiment was carried out at least 3 mo after completion of the insulin clamp experiments. All control experiments were carried out in an identical fashion with infusion of the same amount of insulin solvent, but without administering insulin itself, and with blood sampling performed at the same time intervals including the sampling for blood glucose measurements. To correct for any (nonspecific) enhancement in vascular reactivity caused by the volume expansion due to the infusion of dextrose $20 \%$ to maintain euglycemia (24), a corresponding amount of water, which has a similar volume of distribution as dextrose $20 \%$, was given orally each half hour. Absorption of water from the gastrointestinal tract is rapid and complete so that no relevant lag in volume expansion was to be expected when compared with intravenous dextrose $20 \%$ administration. Because one cannot determine beforehand the amount of dextrose $20 \%$ infused each hour, all control experiments had to be performed after its corresponding insulin clamp experiment. The interval of 3 mo was chosen to exclude any carryover effect. Because the sensitivity to each pressor hormone was again determined three times, on the same moments of the day as during the insulin clamp experiment, correction for any circadian variation of the cardiovascular reactivity could be carried out.

Serum concentrations of potassium and urinary concentrations of sodium were determined by standard laboratory methods. Plasma insulin level, PRA (expressed as the amount of angiotensin I (AI) generated in $1 \mathrm{~h}$ at $4^{\circ} \mathrm{C}$ ), and plasma Ald concentration were measured by radioimmunoassay. Plasma NA and Adr levels were determined by HPLC with electrochemical detection (25).

A quantitative estimate of the insulin sensitivity during the euglycemic clamp was provided by the mean glucose infusion rate determined in each separate hour of the clamp.

Statistical analysis. All results obtained at each test originate from the same nine subjects. To avoid bias due to a possible circadian variation of the variables under evaluation, the measurements obtained during the control experiments were subtracted from the corresponding measurements on the day that also insulin was administered. For each 

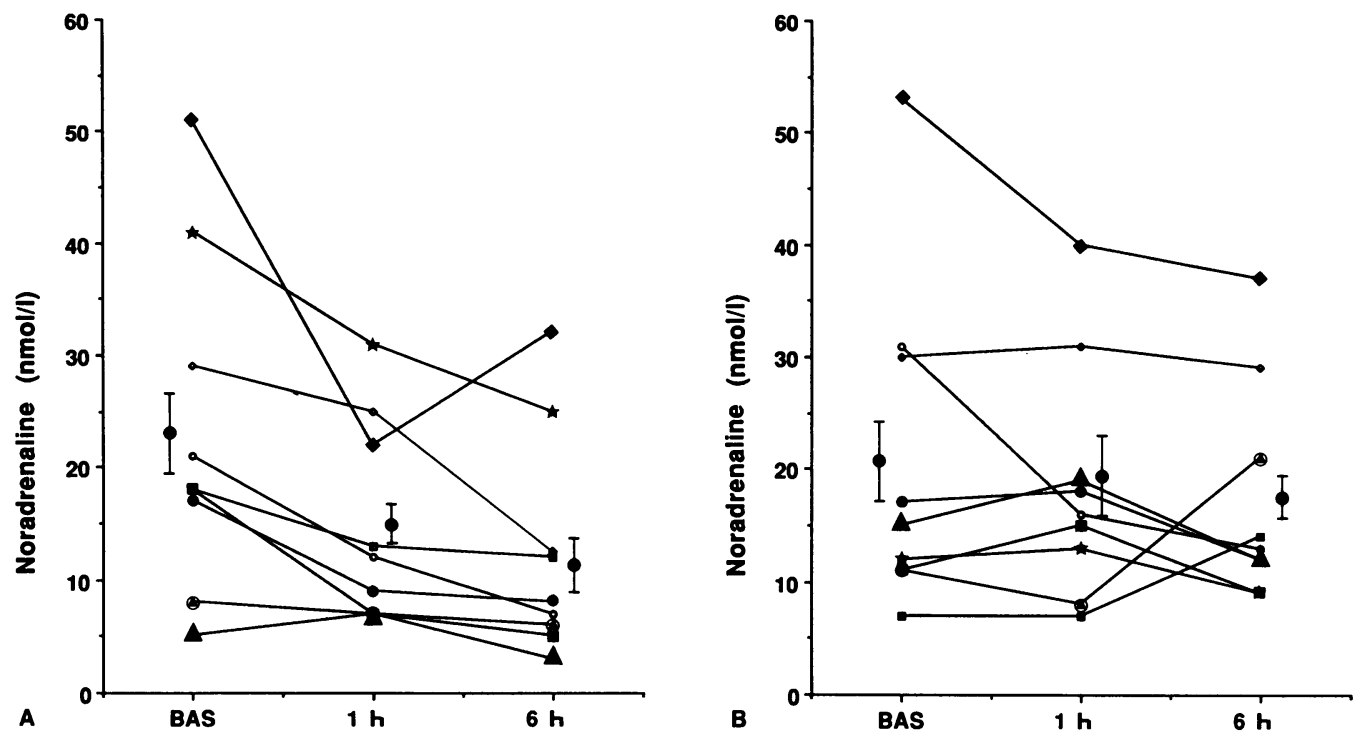

Figure 1. The effect of insulin on the individual changes in circulating noradrenaline levels necessary to increase diastolic blood pressure 20 $\mathrm{mmHg}$ ( $A$, actual experiment; $B$, control experiment; similar symbols have been used for each individual).

variable separately, the adjusted measurements were analyzed by the method of analysis of variance (ANOVA) for repeated measurements. When this analysis yielded significant differences over time, pairwise comparisons were performed using paired $t$ tests. Because the data from the pressor responses were clearly not normally distributed, these results were analyzed using Wilcoxon's signed-rank tests (after the measurements obtained during the control experiments had been subtracted). $24 \mathrm{~h}$ urinary sodium excretion (the average of the last $2 \mathrm{~d}$ ) and plasma insulin levels were also compared with the Wilcoxon's signedrank test. The infused doses of NA were analyzed by the method of analysis of covariance to control for differences in plasma levels. A $P$ value of $<0.05$ was considered significant. Data are reported as mean \pm SEM.

\section{Results}

All subjects showed a fair compliance with the diet. The average $24 \mathrm{~h}$ urinary sodium excretion rate preceding the actual experiment or its respective control, did not differ from each other (AII infusion: $203 \pm 16$ and $198 \pm 17 \mathrm{mmol}$, respectively; NA infusion: $208 \pm 11$ and $192 \pm 9 \mathrm{mmol}$ ). Plasma insulin levels showed comparable increases over basal (Bas) values on the day of AII infusion (Bas, $9 \pm 0.6 ; 1 \mathrm{~h}, 53 \pm 2.5$, and $6 \mathrm{~h}, 60 \pm 4.1$ $\mathrm{mU} /$ liter) and the day of NA infusion (Bas, $10 \pm 2.3 ; 1 \mathrm{~h}$, $57 \pm 3.6$; and $6 \mathrm{~h}, 56 \pm 3.2 \mathrm{mU} / \mathrm{liter}$ ), whereas insulin levels after $6 \mathrm{~h}$ of insulin administration did not differ significantly from those after $1 \mathrm{~h}$. The coefficient of variation of the blood glucose levels during the euglycemic hyperinsulinemic clamp ranged from 4.9 to $8.6 \%$ (mean, $7.0 \%$ ) during AII infusion, and from 3.6 to $9.0 \%$ (mean, $6.0 \%$ ) during NA infusion.

The effect of insulin on the pressor doses of NA, and the changes in circulating plasma NA at a NA-induced increase in DBP of $20 \mathrm{mmHg}$, are given in Table I. A significant decrease in the amount of NA necessary to raise DBP by $20 \mathrm{mmHg}$, as calculated from the changes in plasma NA levels, was noted after insulin administration $(22.6 \pm 9.7$ and $39.9 \pm 13.3 \%$ decrease after 1 and $6 \mathrm{~h}$, respectively). The magnitude of decline in the change in circulating NA to reach the $20 \mathrm{mmHg}$ rise in DBP did not differ significantly after 1 and $6 \mathrm{~h}$ of insulin administration. The individual changes are depicted in Fig. 1.
Although the initial decline after $1 \mathrm{~h}$ of insulin infusion in NA-PD, as calculated from the NA infusion rates, was comparable with that observed for the change in circulating NA to increase DBP $20 \mathrm{mmHg}$, the decline in NA-PD was clearly less marked after $6 \mathrm{~h}$ of insulin administration and not differing anymore from the baseline value $(P=0.26)$. The discrepancy between the disappearance of the NA-PD lowering effect of insulin and a still present decline in the amount of circulating NA necessary to raise DBP $20 \mathrm{mmHg}$ after $6 \mathrm{~h}$ of insulin administration, suggested an influence of insulin on the pharmacokinetics of NA (after that a significant decay of NA in the syringe was excluded; data not shown). It appeared that after $6 \mathrm{~h}$ of insulin administration, significantly lower NA plasma levels were achieved during corresponding NA infusion rates when compared with baseline (linear regression equations Bas and Ins $6 \mathrm{~h}: y=0.067 x+3.15$ and $y=0.067 x-1.85$, respectively; $P<0.01)$. Such a difference could not yet be discerned after $1 \mathrm{~h}$ of insulin infusion $(y=0.067 x+2.34)$; see Fig. 2 .

No change in AII-PD was found after insulin administration (Table I). We did observe a circadian rhythm in the cardiovascular reactivity to AII. Thus, during the clamp a significant decline in AII-PD was observed in the late afternoon when compared with the pressor dose obtained at Bas and $1 \mathrm{~h}$, but during the control experiment a similar decline was noted, although it now did not reach statistical significance $(P=0.08)$.

Serum potassium showed a significant decline after the start of the insulin infusion (NA infusion and AII infusion: both $P<0.0005$, ANOVA) (Table II). The decline, however, was comparable after 1 and $6 \mathrm{~h}$ of insulin administration.

PRA showed a significant increase $1 \mathrm{~h}$ after the clamp was started (Table II). After $6 \mathrm{~h}$ of insulin administration, however, PRA level was back at baseline again. Ald level at the beginning of the pressor infusion was only found to differ significantly from baseline after $1 \mathrm{~h}$ of insulin administration on the day of the NA infusion, but not on the day of the AII infusion (Table II). The rise in Ald during the AII infusion was comparable before, and 1 and $6 \mathrm{~h}$ after insulin administration (Table III). In contrast, the normally occurring rise in plasma PRA and Ald 


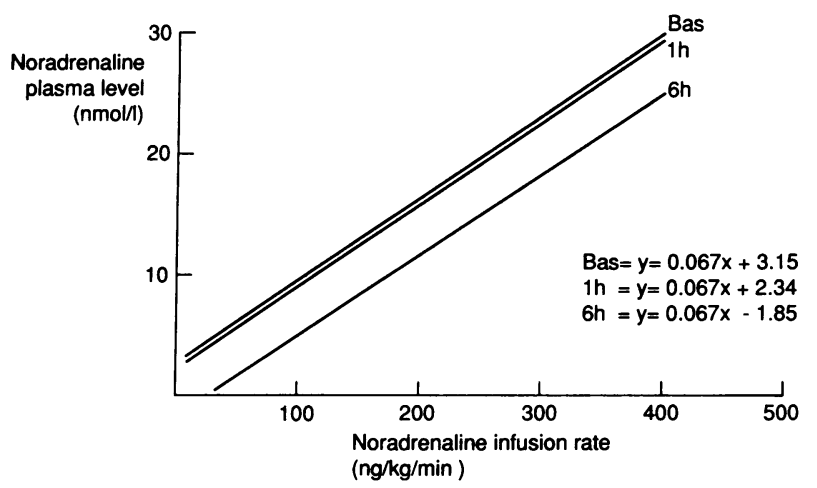

Figure 2. The effect of insulin on the noradrenaline plasma clearance depicted as linear regression equations of the noradrenaline infusion rate with its corresponding plasma level at baseline, and 1 and $6 \mathrm{~h}$ (for explanation see text).

during NA infusion appeared to be abolished during insulin administration (Table IV). Plasma catecholamines levels at the beginning of the pressor infusion did not differ before and after insulin administration, when compared with control values (Table V).

The glucose requirements clearly increased after more prolonged insulin administration (Table VI). The increase in glucose infusion rates on the day of the NA pressor infusions clearly lagged behind that observed on the day of the AII pressor infusions. This is most likely related to the stimulatory effect of NA on gluconeogenesis and not to a difference in insulin sensitivity. No correlation between the change in mean glucose infusion rate and the change in circulating NA to increase DBP $20 \mathrm{mmHg}$ could be discerned.

\section{Discussion}

We have demonstrated that exogenous insulin enhances the cardiovascular responsiveness to NA, as can be derived from the change in circulating plasma NA necessary to increase the diastolic blood pressure $20 \mathrm{mmHg}$. The insulin level at which the increase in cardiovascular reactivity occurred can be consid-

Table I. Effect of Exogenous Insulin on PDs of NA and AII, and Change in Circulating NA Necessary to Increase DBP $20 \mathrm{mmHg}$

\begin{tabular}{lclc}
\hline & \multicolumn{1}{c}{ Bas } & \multicolumn{1}{c}{$1 \mathrm{~h}$} & $6 \mathrm{~h}$ \\
\hline NA $(n g / \mathrm{kg} / \mathrm{min})$ & & & \\
$\quad$ Insulin & $248(93-594)$ & $186(100-380)^{*}$ & $200(114-482)$ \\
$\quad$ Control & $188(99-781)$ & $170(113-555)$ & $189(91-550)$ \\
NA (nmol/liter) & & & \\
$\quad$ Insulin & $18.2(5-51)$ & $11.6(7-31)^{*}$ & $8.4(3-32)^{*}$ \\
$\quad$ Control & $14.9(7-53)$ & $15.9(7-40)$ & $13.3(9-37)$ \\
AII $($ ng/kg/min) & & & \\
$\quad$ Insulin & $23.9(11-75)$ & $19.2(8-108)$ & $16.0(9-62)^{\ddagger}$ \\
Control & $26.3(20-73)$ & $25.0(18-68)$ & $20.8(12-58)^{8}$ \\
& & &
\end{tabular}

Data are given as median and ranges; ${ }^{*} P<0.05,1 \mathrm{~h}$ and/or $6 \mathrm{~h}$ vs. baseline value (Bas); ${ }^{\ddagger} P<0.05,6$ vs. $1 \mathrm{~h}$ and Bas; ${ }^{8} P=0.08,6$ vs. $1 \mathrm{~h}$ and Bas. ered physiologically relevant, because these levels are regularly observed postprandially. The cardiovascular response to AII appeared to be unaltered.

We have to reconcile, however, our findings with the fact that insulin appears to have vasodilating properties at the same time. In animal experiments an attenuation of vasoreactivity after insulin administration has been reported $(10,11)$, although some vascular beds may actually respond with vasoconstriction (10). In man an increase in forearm blood flow has also been demonstrated during infusion of supraphysiological amounts of insulin (26). After infusion of doses within the physiological range, however, no effect of insulin on blood pressure or forearm blood flow could be discerned (27). In accordance, in our study baseline blood pressure at the start of each pressor infusion also did not differ between the actual experiments and their respective controls. It has recently been confirmed that insulin stimulates muscle blood flow in a dose-dependent fashion (28). A nonspecific vasodilation and/or capillary recruitment as a consequence of the increase in tissue metabolism, i.e., glucose oxidation, is thought to underlie that vasodilatory effect $(10,28)$.

A (nonspecific) vasodilatory action of insulin at higher dosages does not, however, preclude any enhancement of the cardiovascular reactivity to vasoactive hormones. The various actions of insulin appear to be largely independent of one another $(8,29)$. For instance, stimulation of the glucose metabolism by insulin in deep forearm tissues of healthy subjects is not coupled with the effect on the $\mathrm{Na} / \mathrm{K}$ exchange (29). If this observation is extrapolated to include other transmembranous cation exchange systems which may be involved in vascular smooth muscle contractility (see below), the lack of an effect of the increase in insulin sensitivity, i.e., glucose oxidation, on the cardiovascular reactivity to NA observed in our study can be understood.

Studies regarding the effect of insulin on cardiovascular reactivity in man are scarce and only the effect on AII responsiveness has been assessed. In accordance with our results no effect was discerned in healthy man (15), whereas in a group of noninsulin-dependent diabetics an attenuation of the pressor response to AII after insulin has been reported (30). The patients in the latter study, however, were in poor metabolic control, which is likely to have confounded the results. Observations in animals do support an interaction between insulin and vasoactive hormones. In the dog, a dose-dependent augmentation of the blood pressure response to AII after insulin administration was observed (13). Also in the dog, the expected decrease in blood pressure, which occurred during infusion of insulin alone, did not take place during chronic NA infusion (14).

The discrepancy between the effect of insulin on the reactivity to NA and to AII is not entirely unexpected. One of the unique features of the increased vascular sensitivity characteristic of the hypertensive state is that it is not the same for all stimuli (31). Thus, the increased cardiovascular responsiveness to NA in essential hypertension can be normalized by potassium supplementation or calcium reentry blockade in contrast to that to AII $(3,32)$. The pathophysiological mechanism underlying that difference, however, remains to be elucidated.

It should be noted that vascular reactivity assessed by blood pressure response is a rather crude index of arteriolar responsiveness. It is the net result of complex actions: e.g., variable amounts of vasoconstriction will take place in different vascu- 
Table II. Effect of Exogenous Insulin on PRA, Ald, and Serum Potassium (K+) at the Start of Each Pressor Infusion

\begin{tabular}{|c|c|c|c|c|c|c|}
\hline & \multicolumn{2}{|c|}{ PRA } & \multicolumn{2}{|c|}{ Ald } & \multicolumn{2}{|c|}{$\mathbf{K}+$} \\
\hline & Insulin & Control & Insulin & Control & Insulin & Control \\
\hline \multicolumn{7}{|c|}{ NA infusion } \\
\hline Bas & $2.4 \pm 0.4$ & $2.0 \pm 0.5$ & $0.21 \pm 0.03$ & $0.18 \pm 0.02$ & $4.2 \pm 0.06$ & $4.1 \pm 0.06$ \\
\hline $1 \mathrm{~h}$ & $4.3 \pm 1.0^{*}$ & $2.2 \pm 0.5$ & $0.30 \pm 0.05^{\ddagger}$ & $0.15 \pm 0.03$ & $3.9 \pm 0.04^{\ddagger}$ & $4.2 \pm 0.08$ \\
\hline $6 \mathrm{~h}$ & $2.7 \pm 0.6$ & $1.8 \pm 0.3$ & $0.14 \pm 0.02$ & $0.08 \pm 0.02$ & $3.7 \pm 0.06^{\ddagger}$ & $4.0 \pm 0.07$ \\
\hline \multicolumn{7}{|c|}{ AII infusion } \\
\hline Bas & $1.6 \pm 0.2$ & $2.5 \pm 0.5$ & $0.20 \pm 0.04$ & $0.34 \pm 0.05$ & $4.2 \pm 0.15$ & $4.1 \pm 0.15$ \\
\hline $1 \mathrm{~h}$ & $2.7 \pm 0.3^{*}$ & $2.6 \pm 0.5$ & $0.27 \pm 0.03$ & $0.43 \pm 0.04$ & $3.9 \pm 0.04^{\ddagger}$ & $4.2 \pm 0.08$ \\
\hline $6 \mathrm{~h}$ & $1.8 \pm 0.6$ & $2.1 \pm 0.4$ & $0.14 \pm 0.03$ & $0.24 \pm 0.04$ & $3.7 \pm 0.06^{*}$ & $4.0 \pm 0.07$ \\
\hline
\end{tabular}

${ }^{*} P<0.05,1 \mathrm{~h}$ and/or $6 \mathrm{~h}$ vs. Bas; ${ }^{\ddagger} P<0.01,1 \mathrm{~h}$ and/or $6 \mathrm{~h}$ vs. Bas.

lar beds (including the venous system), and both direct and reflex effects will exert an influence on cardiac output. Any of these mechanisms may therefore be involved in the enhancement of the cardiovascular reactivity to NA by insulin.

A direct effect of insulin on the vascular smooth muscle cell itself should be considered. Insulin is known to affect several transmembranous cation exchange systems including Na-KATPase, Na-H antiporter, and Ca-Mg-ATPase (33). Stimulation of the Na-K-ATPase will hyperpolarize the cell membrane, making the cell less responsive to stimuli (19). On the other hand, if results obtained in isolated rat adipocytes may be extrapolated to the vascular smooth muscle cell, an increase in intracellular free calcium concentration may occur, thereby potentiating contractility (34). Noteworthy, physiological hyperinsulinemia, which did not lead to any acute hemodynamic changes, has been shown to increase forearm arterial proton release, as evidence of stimulation of the $\mathrm{Na}-\mathrm{H}$ antiport, which in turn was directly related to forearm vascular resistance (27).

Several in vitro studies suggest, however, that insulin may in fact attenuate the vascular responsiveness to NA $(10,35)$, possibly by enhancing the ability of tissue to take up NA (36). Furthermore, studies in vitro and in animals suggest that insulin has chronotropic and inotropic properties itself, but that this action does not dependent on changes in catecholamines $(36,37)$. Whether the observation of an increased ability of tissues to take up NA after insulin administration bears any relation with the observed increase in the plasma clearance of NA in our study, is unclear. An increase in NA clearance has been reported earlier (38), although not consistently (39). It does, however, readily explain why the initial decline in NA

Table III. Effect of Insulin on AII-stimulated Plasma Ald Levels (nmol/liter)

\begin{tabular}{cccccccc}
\hline & \multicolumn{3}{c}{ Insulin } & & \multicolumn{3}{c}{ Control } \\
\cline { 2 - 3 } \cline { 6 - 8 } \cline { 6 - 8 } & Bas & $1 \mathrm{~h}$ & $6 \mathrm{~h}$ & & Bas & $1 \mathrm{~h}$ & $6 \mathrm{~h}$ \\
\hline O & $0.20 \pm 0.04$ & $0.27 \pm 0.03$ & $0.14 \pm 0.03$ & & $0.34 \pm 0.05$ & $0.43 \pm 0.04$ & $0.24 \pm 0.04$ \\
I & $0.62 \pm 0.08$ & $0.60 \pm 0.13$ & $0.69 \pm 0.09$ & & $0.67 \pm 0.06$ & $0.74 \pm 0.10$ & $0.58 \pm 0.05$ \\
II & $0.95 \pm 0.15$ & $0.89 \pm 0.12$ & $1.02 \pm 0.14$ & & $0.96 \pm 0.10$ & $0.94 \pm 0.12$ & $0.91 \pm 0.27$
\end{tabular}

$O$, at the beginning of each pressor infusion; I, at a rise in $\mathrm{DBP}$ of $\sim 10 \mathrm{mmHg}$, II, at a rise in DBP of $\sim 20 \mathrm{mmHg}$. pressor dose, as calculated from the NA infusion rates, returned to baseline again after more prolonged insulin infusion.

Considering the rise in PRA over basal values after insulin administration, an increase in AII could be an attractive alternative explanation for the observed increase in cardiovascular responsiveness to NA. Even at subpressor doses, AII has been found to potentiate the cardiovascular response to NA in both normotensive and hypertensive man (40). This hypothesis seems, however, less tenable because PRA levels returned back to baseline after $6 \mathrm{~h}$ of insulin infusion. On the other hand, converting enzyme inhibition has recently been shown to attenuate the increased cardiovascular reactivity in patients with essential hypertension, although not in patients with diabetes (41). If one assumes that also AII levels were elevated by exogenous insulin, the absence of a change in AII pressor dose may in fact signify an increase in cardiovascular reactivity to this hormone, as alterations in plasma levels of vasoactive substances clearly affect the pressor responsiveness to these substances and a negative correlation between the two has been reported $(2,42)$.

Volume expansion itself is an unlikely explanation for the increase in vascular responsiveness to NA (24). To correct for any change in pressor response during the clamp as a consequence of the infusion of dextrose $20 \%$ to maintain euglycemia, all subjects received corresponding amounts of water orally on the day of the control experiment. Moreover, any change in cardiovascular reactivity would have been expected to affect the pressor response to NA and AII equally (24). The

Table IV. Effect of Insulin on NA-stimulated Plasma Ald Level and PRA (Data of Control Studies Omitted)

\begin{tabular}{|c|c|c|c|c|c|c|}
\hline & \multicolumn{3}{|c|}{ Ald } & \multicolumn{3}{|c|}{ PRA } \\
\hline & Bas & $1 \mathrm{~h}$ & $6 \mathrm{~h}$ & Bas & $1 \mathrm{~h}$ & $6 \mathrm{~h}$ \\
\hline & nmol/liter & nmol/liter & nmol/liter & $n g A l / m l / h$ & $n g A l / m l / h$ & $n g A l / m l / h$ \\
\hline o & $0.21 \pm 0.03$ & $0.30 \pm 0.05$ & $0.14 \pm 0.02$ & $2.4 \pm 0.4$ & $4.3 \pm 1.0^{*}$ & $2.7 \pm 0.6$ \\
\hline I & $0.49 \pm 0.07$ & $0.28 \pm 0.06^{*}$ & $0.15 \pm 0.04^{*}$ & $4.1 \pm 0.7$ & $4.1 \pm 1.0$ & $2.7 \pm 0.6$ \\
\hline II & $0.63 \pm 0.09$ & $0.28 \pm 0.06^{*}$ & $0.20 \pm 0.07^{*}$ & $4.7 \pm 0.8$ & $3.8 \pm 0.7^{*}$ & $2.6 \pm 0.5^{*}$ \\
\hline
\end{tabular}

$\mathrm{O}$, at the beginning of each pressor infusion; $\mathrm{I}$, at a rise in DBP of $\sim 10 \mathrm{mmHg}$; II, at a rise in DBP of $\sim 20 \mathrm{mmHg}$.

${ }^{*} P<0.05,1$ and/or $6 \mathrm{~h}$ vs. Bas) 
Table V. Effect of Exogenous Insulin on Plasma Catecholamines at the Start of Each Pressor Infusion

\begin{tabular}{lcclll}
\hline & \multicolumn{2}{c}{ NA } & & \multicolumn{2}{c}{ Adr } \\
\cline { 2 - 3 } \cline { 5 - 6 } & Insulin & Control & & Insulin & Control \\
\hline NA infusion & & & & \\
Bas & & & & & \\
1 h & $1.34 \pm 0.14$ & $0.83 \pm 0.09$ & & $0.38 \pm 0.05$ & $0.34 \pm 0.04$ \\
6 h & $0.91 \pm 0.14$ & $0.97 \pm 0.12$ & & $0.34 \pm 0.05$ & $0.39 \pm 0.05$ \\
AII infusion & & & & \\
Bas & $0.82 \pm 0.14$ & $1.04 \pm 0.09$ & & $0.37 \pm 0.05$ & $0.40 \pm 0.05$ \\
1 h & $0.89 \pm 0.11$ & $1.14 \pm 0.12$ & & $0.37 \pm 0.05$ & $0.41 \pm 0.05$ \\
$6 \mathrm{~h}$ & $0.79 \pm 0.14$ & $1.03 \pm 0.09$ & & $0.42 \pm 0.05$ & $0.41 \pm 0.04$ \\
& & & & & \\
\hline
\end{tabular}

same holds true for any volume expansion due to sodium retention as a result of the renal tubular action of insulin $(43,44)$.

The increase in cardiovascular reactivity to AII during the day is in accordance with the circadian rhythm of the RAAS (45). At the end of the day PRA and AII levels reach their nadir and, as a consequence, an increase in cardiovascular reactivity is to be expected (42). The failure to reach statistical significance on the day of the control experiment is most likely due to a type II error. No such clearcut variation in cardiovascular reactivity was demonstrated for NA. This agrees with another study, in which three consecutive infusions with NA were performed and found not to differ (46). This does not imply that a circadian rhythm of this hormone does not exist. Plasma catecholamines are significantly higher during day time than at night, reaching their highest levels late in the morning (47; see also Table V). As shown in Fig. $1 B$ (concerning the control experiments), the cardiovascular reactivity to NA tended to increase slightly at $6 \mathrm{~h}$ (i.e., late in the afternoon when NA levels are declining again) in several volunteers. The presence of a circadian rhythm in cardiovascular reactivity points at the same time to the necessity of adequate control studies whenever consecutive studies are performed; two of the studies mentioned above are in lack of this $(15,40)$.

In accordance with previous reports, divergent effects of insulin on the renin angiotensin aldosterone system (RAAS) were noted $(44,48)$. The observed increase in PRA without a

Table VI. Effect of Duration of Insulin Administration on Insulin Sensitivity Denoted by Mean Glucose Infusion Rate

\begin{tabular}{ccr}
\hline & \multicolumn{2}{c}{ Glucose infusion rate } \\
\cline { 2 - 3 } $\begin{array}{c}\text { Duration of } \\
\text { insulin administration }\end{array}$ & $\begin{array}{c}\text { NA/insulin } \\
\text { clamp experiment }\end{array}$ & $\begin{array}{c}\text { AlI/insulin } \\
\text { clamp experiment }\end{array}$ \\
\hline min & $\mathrm{mg} \cdot \mathrm{kg}^{-1} \cdot \mathrm{min}^{-1}$ & $\mathrm{mg} \cdot \mathrm{kg}^{-1} \cdot \mathrm{min}^{-1}$ \\
60 & $5.1 \pm 0.7$ & $6.6 \pm 1.0$ \\
120 & $5.3 \pm 0.8$ & $8.7 \pm 0.8$ \\
180 & $5.9 \pm 0.8$ & $8.9 \pm 0.8$ \\
240 & $7.8 \pm 0.7$ & $9.4 \pm 1.0$ \\
300 & $8.2 \pm 1.0$ & $9.8 \pm 0.9$ \\
360 & $9.4 \pm 0.7$ & $9.5 \pm 0.8$ \\
420 & $9.5 \pm 0.9$ & $11.2 \pm 0.7$
\end{tabular}

concomitant rise in plasma Ald has been ascribed to the insulin-induced decrease in serum potassium concentration, because no changes occurred when the fall in plasma potassium was prevented (48). However, PRA declined at the end of the clamp despite persistence of hypokalemia, suggesting that some other counterregulatory mechanism comes into play. We did not observe an amelioration of the decline in potassium after more prolonged insulin administration, as suggested previously (20). Also, no stimulatory effects of NA on the RAAS was noted anymore after the infusion of insulin was started. The AII-induced stimulation of aldosterone secretion was intact, although we could not confirm earlier observations of an enhancement of the AII-stimulated aldosterone secretion (15). As reported previously, we could not discern any stimulatory effect of insulin on circulating plasma noradrenaline levels as an index of the sympathetic nervous system activity when compared with control levels (44).

In conclusion, we have demonstrated an increase in cardiovascular responsiveness to NA during concomitant administration of insulin at a physiological dose. It remains to be proven whether this contributes to the elevation in blood pressure that is frequently observed in patients with insulin resistance and, as a consequence, hyperinsulinemia, e.g., patients with essential hypertension or noninsulin-dependent diabetes mellitus. If insulin is to contribute to the elevated blood pressure in insulinresistant states, then a persistent sensitivity for the cardiovascular (and/or renal) action(s) of insulin has to be postulated despite resistance to the glucose lowering action at the same time. Preliminary results in patients with NIDDM suggest that this may actually be the case. These patients appear to be still sensitive to the antinatriuretic action of insulin (unpublished observations).

\section{Acknowledgments}

This study was supported by grant C 87.720 and C 89.865 of the Dutch Kidney Foundation (Nier Stichting Nederland).

\section{References}

1. Goldenberg, M., K. L. Pines, E. Baldwin, D. G. Greene, and C. Roh. 1948. The hemodynamic response of man to norepinephrine and epinephrine and its relation to the problem of hypertension. Am. J. Med. 5:792-806.

2. Meier, A., P. Weidmann, M. Grimm, G. Keusch, Z. Gluck, and I. Minder 1981. Pressor factors and cardiovascular pressor reactivity in borderline essential hypertension. Hypertension (Dallas). 3:367-372.

3. Bianchetti, M. G., P. Weidmann, C. Beretta-Piccoli, and C. Ferrier. 1987. Potassium and norepinephrine- or angiotensin-mediated pressor control in prehypertension. Kidney Int. 31:956-963.

4. Lenders, J. W. M., T. De Boo, W. A. J. Lemmens, J. J. Willemsen, and T. Thien. 1989. Cardiovascular responsiveness to norepinephrine in mild essential hypertension. Am. J. Cardiol. 63:1231-1234.

5. Bianchetti, M. G., C. Beretta-Piccoli, P. Weidmann, and C. Ferrier. 1986. Blood pressure control in normotensive members of hypertensive families. Kidney Int. 29:882-888.

6. Lais, L. T., and M. J. Brody. 1978. Vasoconstrictor hyperresponsiveness: an early pathogenetic mechanism in the spontaneously hypertensive rat. Eur. $J$. Pharmacol. 47:177-189.

7. Mulvany, M. J., C. Aalkjaer, and J. Christensen. 1980. Changes in noradrenaline sensitivity and morphology of arterial resistance vessels during the development of high blood pressure in spontaneously hypertensive rats. Hypertension (Dallas). 2:664-671.

8. Ferrannini, A. M., G. Buzzigoli, R. Bonadonna, M. A. Giorico, M. Oleggini, L. Graziadei, R. Pedrinelli, L. Brandi, and S. Bevilacqua. 1987. Insulin resistance in essential hypertension. $N$. Engl. J. Med. 317:350-357. 
9. Fournier, A. M., M. T. Gadia, D. B. Kubrusly, J. S. Skyter, and J. M. Sosenko. 1986. Blood pressure, insulin and glycemia in non-diabetic subjects. Am. J. Med. 83:861-864.

10. Liang, C. S., J. U. Doherty, R. Faillace, K. Maekawa, S. Arnold, H. Gavras, and W. B. Hood. 1982. Insulin infusion in conscious dogs. Effects on systemic and coronary hemodynamics, regional blood flows, and catecholamines. J. Clin. Invest. 69:1321-1336.

11. Yagi, S., S. Takata, H. Kiyok, M. Yamamoto, Y. Noto, T. Ikeda, and N. Hattori. 1988. Effects of insulin on vasoconstrictive responses to norepinephrine and angiotensin II in rabbit femoral artery and vein. Diabetes. 37:1064-1067.

12. Hall, J. E., T. G. Coleman, H. L. Mizell, and M. J. Smith, Jr. 1990 Chronic hyperinsulinemia and blood pressure regulation. Am. J. Physiol. 258:F722-F731.

13. Rocchini, A. P., C. Moorehead, S. DeRemer, T. L. Goodfriend, and D. L. Ball. 1990. Hyperinsulinemia and the aldosterone and pressor responses to angiotensin II. Hypertension (Dallas). 15:861-866.

14. Hall, J. E., M. W. Brands, S. D. Kivlighn, H. L. Mizelle, D. A. Hildebrandt, and C. A. Gaillard. 1990. Chronic hyperinsulinemia and blood pressure. Interaction with catecholamines. Hypertension (Dallas). 15:519-527.

15. Vierhapper, H., W. Waldhausl, and P. Nowotny. 1983. The effect of insulin on the rise in blood pressure and plasma aldosterone after angiotensin II in normal man. Clin. Sci. 64:383-386.

16. Weidmann, P., C. Beretta-Piccoli, G. Keusch, Z. Gluck, M. Mujagic, M. Grimm, A. Meier, and W. H. Ziegler. 1979. Sodium-volume factor, cardiovascular reactivity and hypotensive mechanism of diuretic therapy in mild hypertension associated with diabetes mellitus. Am. J. Med. 67:779-784.

17. Abumrad, N., D. Rabin, M. Diamond, and W. Lacy. 1981. Use of heated superficial hand vein as alternative site for the measurement of aminoacid concentrations and for the study of glucose and alanine kinetics. Metabolism. 30:936-940.

18. Ponchner, M., R. J. Heine, A. Pernet, I. Hanning, A. J. Francis, D. Cook, H. Orskov, and K. G. M. M. Alberti. 1984. A comparison of the artificial pancreas (glucose controlled insulin infusion system) and a manual technique for assessing insulin sensitivity during euglycaemic clamping. Diabetologia. 26:420 425.

19. Iannaccone, S. T., L. Kai-Xun, N. Sperelakis, and D. A. Lathrop. 1989. Insulin-induced hyperpolarization in mammalian skeletal muscle. Am. J. Physiol. 256:C368-C374.

20. Minaker, K. L., and J. W. Rowe. 1982. Potassium homeostasis during hyperinsulinemia: effect of insulin level, $\beta$-blockade, and age. Am. J. Physiol. 242:E373-E377.

21. Doberne, L., M. S. Greenfield, B. Schulz, and G. M. Reaven. 1981. Enhanced glucose utilization during prolonged glucose clamp studies. Diabetes. 30:829-835.

22. Chinn, R. H., and G. Dusterdieck. 1972. The response of blood pressure to infusion of angiotensin II: relation of blood pressure to plasma concentrations of renin and angiotensin II. Clin. Sci. 42:489-504.

23. Distler, A., Ch. Barth, H. Liebau, P. Vecsei, and H. P. Wolff. 1970. The effect of tyramine, noradrenaline, and angiotensin on the blood pressure in hypertensive patients with aldosteronism and low plasma renin. Eur. J. Clin. Invest. 1:196-203.

24. Otsuka, A., T. Ogihara, K. Kohara, H. Mikami, K. Katahira, T. Tsunetoshi, and Y. Kumahara. 1988. Vasoconstriction and hypersensitivity to vasoactive substances after acute volume expansion in dogs. Hypertension (Dallas). 12:5966.

25. Popp-Snijders, C., B. Geenen, and E. A. P. van der Heijden. 1989. Serum noradrenaline is composed of plasma and platelet noradrenaline. Ann. Clin. Bio chem. 26:191-192.

26. Scott, A. R., T. Bennett, and I. A. Macdonald. 1988. Effects of hyperinsulinaemia on the cardiovascular responses to graded hypovolaemia in normal and diabetic subjects. Clin. Sci. 75:85-92.

27. Natali, A., G. Buzzigoli, S. Taddei, D. Santori, M. Cerri, R. Pedrinelli, and
E. Ferrannini. 1990. Effects of insulin on hemodynamics and metabolism in human forearm. Diabetes. 39:490-500.

28. Laakso, M., S. V. Edelman, and A. D. Baron. 1990. Decreased effect of insulin to stimulate skeletal muscle blood flow in obese man. J. Clin. Invest. 85:1844-1852.

29. Ferrannini, E., S. Taddei, D. Santorro, A. Natali, C. Boni, D. Del Chiaro, and G. Buzzigoli. 1988. Independent stimulation of glucose metabolism and $\mathrm{Na} / \mathrm{K}$ exchange by insulin in the human forearm. Am. J. Physiol. 255:E953E958.

30. Yamamoto, Y., S. Takata, S. Yagi, N. Iwasi, H. Kiyokawa, Y. Noto, T. Ikeda, and N. Hattori. 1986. Effects of insulin on pressor responsiveness and baroreflex function in diabetes mellitus. Jpn. Circ. J. 50:943-948.

31. Bohr, D. F., and R. C. Webb. 1988. Vascular smooth muscle membrane in hypertension. Annu. Rev. Pharmacol. Toxicol. 28:389-409.

32. Bianchetti, M. G., C. Beretta-Piccoli, P. Weidmann, L. Link, K. Boehringer, C. Ferrier, and J. J. Morton. 1983. Calcium and blood pressure regulation in normal and hypertensive subjects. Hypertension (Dallas). 5(Suppl. II):57-65.

33. Moore, R. D. 1983. Effects of insulin upon ion transport. Biochim. Biophys. Acta. 737:1-49.

34. Draznin, B., M. Koa, and K. E. Sussman. 1987. Insulin and glyburide increase cystolic free-Ca concentration in isolated rat adipocytes. Diabetes. 36:174-178.

35. Alexander, W. D., and R. J. Oake. 1977. The effect of insulin on vascular reactivity to norepinephrine. Diabetes. 26:611-614.

36. Bhagat, B., W. J. Burke, and N. S. Dhalla. 1981. Insulin-induced enhancement of uptake of noradrenaline in atrial strips. Br. J. Pharmacol. 74:325-332.

37. Lee, J. C., and J. E. Downing. 1976. Effects of insulin on cardiac muscle contraction and responsiveness to norepinephrine. Am. J. Physiol. 230:13601365 .

38. Danforth, E., K. J. Acheson, L. Christin, E. Ravussin, R. L. Galeazzi, and E. Jequier. 1987. A role for the sympathetic nervous system in the regulation of resting and glucose/insulin-stimulated thermogenesis in man. Clin. Res. 35:501A. (Abstr.)

39. Rowe, J. W., J. B. Young K. L. Minaker, A. L. Stevens, J. Pallotta, and L. Landsberg. 1981. Effect of insulin and glucose infusions on sympathetic nervous system activity in normal man. Diabetes. 30:219-225.

40. Reams, G. P., and J. H. Bauer. 1987. Angiotensin II potentiates the vasoconstrictive effect of norepinephrine in normotensive and hypertensive man. $J$. Clin. Hypertens. 3:610-616.

41. Strocchi, E., P. L. Malini, A. Ciavarella, G. Valtancoli, C. Ricci, A. Mustacchio, and E. Ambrosioni. 1990. Pressor responsiveness to noradrenaline in diabetic patients and ACE-inhibition. Am. J. Hypertens. 3:118A. (Abstr.)

42. Kaplan, N. M., and J. G. Silah. 1964. The effect of angiotensin II on the blood pressure in humans with hypertensive disease. J Clin. Invest. 43:659-669.

43. DeFronzo, R. A., C. R. Cooke, R. Andres, G. R. Faloona, and P. J. Davis. 1975. The effects of insulin on renal handling of sodium, potassium, calcium, and phosphate in man. J. Clin. Invest. 55:845-855.

44. Gans, R. O. B., L. v.d. Toorn, H. J. G. Bilo, J. J. P. Nauta, R. J. Heine, and A. J. M. Donker. 1991. Renal and cardiovascular effects of exogenous insulin in healthy volunteers. Clin. Sci. 80:219-225.

45. Modlinger, R. S., K. Sharif-Zadeh, N. H. Ertel, and M. Gutkin. 1976. The circadian rhythm of renin. J. Clin. Endocrinol. Metab. 43:1276-1282.

46. Kurpad, A. V., R. N. Kulkarni, M. Vaz, and P. S. Shetty. 1989. Repeated infusions of identical doses of norepinephrine show potentiation of metabolic responses in human subjects. Metabolism. 38:979-982.

47. Turton, M. D., and T. Deegan. 1974. Circadian variation of plasma catecholamine, cortisol and immunoreactive insulin concentration in supine subjects. Clin. Chim. Acta. 55:389-397.

48. Trovati, M., P. Massucco, G. Anfossi, F. Cavalot, E. Mularoni, L. Mattiello, G. Rocca, and G. Emanuelli. 1989. Insulin influences the renin-angiotensin-aldosterone system in humans. Metabolism. 38:501-503. 